\title{
Modeling house price dynamics with heterogeneous speculators
}

\author{
Roberto Dieci \\ Department of Mathematics for Economics and Social Science, University of Bologna \\ Viale Q. Filopanti 5, I-40126 Bologna, Italy \\ Frank Westerhoff \\ Department of Economics, University of Bamberg \\ Feldkirchenstrasse 21, D-96045 Bamberg, Germany
}

February 3, 2012

\begin{abstract}
This paper investigates the impact of speculative behavior on house price dynamics. Speculative demand for housing is modeled using a heterogeneous agent approach, whereas 'real' demand and housing supply are represented in a standard way. Together, real and speculative forces determine excess demand in each period and house price adjustments. Three alternative models are proposed, capturing in different ways the interplay between fundamental trading rules and extrapolative trading rules, resulting in a $2 D$, a $3 D$, and a $4 D$ nonlinear discretetime dynamical system, respectively. While the destabilizing effect of speculative behavior on the model's steady state is proven in general, the three specific cases illustrate a variety of situations that can bring about endogenous dynamics, with lasting and significant price swings around the 'fundamental' price, as we have seen in many real markets.
\end{abstract}

Keywords: Heterogeneous expectations, Housing markets, Boom-bust cycles, Bifurcation analysis

JEL Classification: C62, D84, R21, R31

\section{Introduction}

The recent Global Financial and Economic Crisis was essentially triggered by the dramatic collapse of the US housing market. However, significant boom-bust housing price cycles have occurred for centuries in many countries around the world. ${ }^{1}$ Shiller $(2005,2008)$ argues that no specific factors are responsible for such price movements but that something broad and general is at work in these markets. In his opinion, it is the speculative behavior of the market participants that repeatedly leads to stunning price movements in housing markets. Unfortunately, not many theoretical approaches exist which take up this theme (some rare exceptions include Piazzesi and Schneider 2009, Leung et al. 2009, Dieci and Westerhoff 2011, Kouwenberg and Zwinkels 2011). In this paper, we

\footnotetext{
${ }^{1}$ For historical accounts and empirical evidence, see Eichholtz (1997), Eitrheim and Erlandsen (2004), Kindleberger and Aliber (2005), Shiller (2005) and Case (2010), amongst others.
} 
thus seek to develop a simple framework in which speculative demand for houses is influenced by heterogeneous expectations. By showing how such behavior may trigger irregular boom-bust housing price dynamics, we hope to be able to improve our understanding of housing market dynamics. Since housing market crashes may be quite harmful for the real economy, we consider this particularly important.

In a nutshell, the structure of our model is as follows. Housing prices change in response to the misbalance between the demand for and the supply of houses (both to be interpreted as flow variables). Housing demand consists of two components. First, there is a real demand for houses, which decreases with the current housing price. Second, there is a speculative demand, which depends (positively or negatively) on the current and the last $L$ observed housing prices. The supply of houses is also made up of two components. On the one hand, new houses are constructed in every period, where the amount of new houses is positively related to the current housing price. On the other hand, a certain (constant) fraction of the existing stock of houses enters the housing market. Of course, the stock of houses evolves over time with respect to new housing construction and the depreciation of the existing stock of houses.

Using a mixture of analytical and numerical tools, we derive the following results. In the absence of speculation, the price of houses and the stock of houses are driven by a two-dimensional map. There is a unique steady state which is locally stable as long as the slope of the demand for houses, the price sensitivity of new housing construction and the fraction of the stock of houses ready for sale are not too high. In order to appreciate the effect of speculation on the dynamics of housing markets, we assume that this condition is always fulfilled. The dynamics of the complete model is due to an $(L+2)$-dimensional map and it is possible to show that speculation may destabilize an otherwise stable housing market. However, to gain a clearer picture of what may trigger boomand-bust housing price dynamics, we consider three particular cases of speculative demand. ${ }^{2}$

In the first setup, there are two types of speculators. Extrapolators believe in the persistence of bull and bear market dynamics. Therefore, their demand is positive if there is a bull market and negative if there is a bear market. In contrast, fundamentalists buy houses if the market is undervalued and sell houses if it is overvalued - believing that prices will eventually return towards their fundamentals. Both demand functions are linear. What makes the model nonlinear is that the market fractions of these two groups vary over time. In the first setup, we assume that speculative demand is based more heavily on the fundamental rule as the price disconnects from the fundamental value. As it turns out, the dynamics is still driven by a two-dimensional map. However, the fundamental steady state may now lose stability due to either a pitchfork bifurcation or a Neimark-Sacker bifurcation. Simulations reveal that both scenarios can lead to interesting housing market dynamics where there are lasting, significant and complicated swings in housing prices.

In our second setup, we keep the market fractions of chartists and fundamentalists constant. In

\footnotetext{
${ }^{2}$ These demand specifications are heavily inspired by recent work in agent-based financial market modeling in which chartists interact with fundamentalists, as surveyed in Chiarella et al. (2009), Hommes and Wagener (2009), Lux (2009) and Westerhoff (2009). Laura Gardini contributed to this research area quite substantially, see, e.g. Chiarella et al. (2002, 2005), Bischi et al. (2006) and Tramontana et al. (2009), to name only a few of her works. It is typically Laura who miraculously accomplishes an otherwise "undoable" mathematical analysis.
} 
addition, chartists now explicitly extrapolate past price changes. Their demand is positive if the last observed house price change is positive, and vice versa. However, the demand function of the chartists is $S$-shaped. Fundamentalists, in turn, still speculate on a fundamental price correction. Now the dynamics of the model is driven by a three-dimensional map, and there is only a unique fundamental steady state. Here we find analytical and numerical evidence of a Neimark-Sacker bifurcation, endogenous housing price dynamics and the coexistence of attractors.

In our third setup, chartists (again) extrapolate the most recent price trend, yet once again their rule is linear. Fundamentalists also rely on a linear rule. Compared to the first setup, speculators now switch between rules with respect to their past fitness, measured by the rules' squared forecasting errors. Clearly, we assume that a rule that produces lower squared forecasting errors than the other rule is preferred by the majority of speculators. Speculators therefore display a form of boundedly rational learning behavior. The dynamical system of our final setup is fourdimensional, and again possesses a unique fundamental steady state. Its local stability may be lost via a Neimark-Sacker bifurcation, and simulations reveal again the emergence of complex endogenous dynamics.

Overall, our paper thus demonstrates that speculative behavior may destabilize housing markets. In all of our setups, endogenous dynamics may set in, typically in connection with a NeimarkSacker bifurcation. These dynamics imply lasting and significant price swings around the fundamental steady state, as we have seen in many real markets. However, in the first setup there is also the possibility of a pitchfork bifurcation and thus of scenarios where there are permanent bull or bear markets. Hence, in the absence of exogenous shocks, a housing market may remain persistently overvalued or undervalued. It is remarkable that similar scenarios can also be found in the second and third setup, although not directly associated with local bifurcations. Moreover, as we will see, endogenous switches between bull and bear markets may occur if speculative behavior is strong enough. There is thus a second, alternative route which leads to endogenous boom-and-bust housing price dynamics.

The remainder of the paper is organized as follows. In Section 2, we present the core of our model and derive some general results. In Section 3, we introduce the three different setups of our model and provide some analytical and numerical results on how speculative housing markets function. In Section 4, we conclude the paper and identify avenues for future research.

\section{The general model setup}

Housing demand and supply in a given period represent flows. Housing demand consists of a real demand component and a speculative demand component. As usual, real demand is expressed as a negatively-sloped function $D(p)$ of housing price $p, D^{\prime}<0$. Speculative demand is expressed, generically, as a function $\Phi$ of current and past prices. Total flow demand in period $t$ is thus given by

$$
D_{t}^{\text {flow }}=D\left(p_{t}\right)+\Phi\left(p_{t}, p_{t-1}, p_{t-2}, \ldots, p_{t-L}\right)
$$


where $p_{t}$ denotes the housing price at the beginning of period $t$, and the integer $L \geq 0$ represents the memory lag in speculators' investment rules and expectations. Housing flow supply consists of new housing and a fraction of the existing housing stock:

$$
S_{t}^{\text {flow }}=I\left(p_{t}\right)+\lambda h_{t},
$$

where $I(p), I^{\prime}>0$, represents new constructions in the period, $0<\lambda<1$, and $h_{t}$ is the stock of housing at the beginning of period $t$. We see from equation (2) that, besides including new housing investments, housing supply in period $t$ also includes a (small) constant fraction of the existing stock of housing, $\lambda h_{t}$. This assumption, which has already been used in the literature on urban economics (see, e.g. Glaeser et al. 2008) ${ }^{3}$, introduces a simple connection between the stock of housing and the amount of existing homes for sale in a given period. ${ }^{4}$

House price changes are proportional to excess demand for houses, $D_{t}^{\text {flow }}-S_{t}^{\text {flow }}$, so that the price at the beginning of period $t+1$ is given by

$$
p_{t+1}=p_{t}+\alpha\left(D\left(p_{t}\right)+\Phi\left(p_{t}, p_{t-1}, p_{t-2}, \ldots, p_{t-L}\right)-I\left(p_{t}\right)-\lambda h_{t}\right)
$$

where $\alpha>0$ is the price adjustment parameter.

Housing stock evolves according to a standard 'asset accumulation' equation due to new investment and depreciation, as follows:

$$
h_{t+1}=(1-\delta) h_{t}+I\left(p_{t}\right)
$$

where $0<\delta<1$ is the housing depreciation rate.

By defining current price and housing stock as $p$ and $h$, respectively, and lagged price variables as $p_{-k}, k=1,2, \ldots, L$, the discrete-time model described by equations (3) and (4) can be represented as the iteration of the $(L+2)$-dimensional map:

$$
\left\{\begin{array}{l}
h^{\prime}=(1-\delta) h+I(p) \\
p^{\prime}=p+\alpha\left(D(p)+\Phi\left(p, p_{-1}, p_{-2}, \ldots, p_{-L}\right)-I(p)-\lambda h\right) \\
p_{-1}^{\prime}=p \\
p_{-2}^{\prime}=p_{-1} \\
\vdots \\
p_{-L}^{\prime}=p_{-L+1}
\end{array}\right.
$$

where here the symbol ' denotes the unit time advancement operator.

\footnotetext{
${ }^{3}$ As discussed in Glaeser et al. (2008), this assumption can be justified in terms of the existence of a continuum of homeowners, receiving a Poisson-distributed shock in each period that forces them to sell their homes and leave the area. Of course, in a more realistic setup, probability $\lambda$ of the shock might itself depend on the current price or on expected price movements.

${ }^{4}$ Thanks to this assumption and the following equation (4), a bidirectional relationship between housing stock and housing supply flow is established.
} 


\subsection{The model in the absence of speculation}

We start by determining the steady state solution in the absence of speculation, thus we first assume $\Phi \equiv 0$. In this case the dynamics of price and stock of housing is in fact driven by the two-dimensional map

$$
\left\{\begin{array}{l}
h^{\prime}=(1-\delta) h+I(p) \\
p^{\prime}=p+\alpha(D(p)-I(p)-\lambda h)
\end{array} .\right.
$$

A stationary point $\left(h^{*}, p^{*}\right)$ needs to satisfy the conditions:

$$
\left\{\begin{array}{l}
\delta h^{*}=I\left(p^{*}\right) \\
D\left(p^{*}\right)=I\left(p^{*}\right)+\lambda h^{*}
\end{array},\right.
$$

from which it follows that

$$
h^{*}=\frac{I\left(p^{*}\right)}{\delta},
$$

where $p^{*}$ is implicitly defined by

$$
\delta D\left(p^{*}\right)-(\lambda+\delta) I\left(p^{*}\right)=0
$$

We assume that a strictly positive solution to (9) exists, satisfying $D\left(p^{*}\right)>0, I\left(p^{*}\right)>0 .{ }^{5}$ This solution is thus necessarily unique due to our general assumptions on functions $D$ and $I$. We call $p^{*}$ the fundamental price and $\left(h^{*}, p^{*}\right)$ the fundamental steady state (FSS henceforth).

It can be shown that under iteration of map (6) the FSS is locally asymptotically stable (LAS henceforth), provided that neither the slopes of the demand and investment functions nor parameter $\lambda$ are too large. Leaving aside the full characterization of the parameter region in which the FSS is LAS, a simple sufficient condition for stability of the model without speculation can easily be derived, under general $D$ and $I$. This 'broad' condition will be assumed to hold in the rest of the paper, in order to focus on the 'unstable' dynamics emerging from speculative behavior. Details are provided below.

The Jacobian matrix (at the FSS) of the 2-D map (6) is:

$$
J:=\left(\begin{array}{cc}
1-\delta & I^{\prime} \\
-\alpha \lambda & 1+\alpha\left(D^{\prime}-I^{\prime}\right)
\end{array}\right),
$$

where the derivatives ${ }^{6}$ of $D$ and $I$ are computed at the fundamental price $p^{*}$. A necessary and sufficient condition on the parameters $\alpha, \delta, \lambda$ and on the slopes $D^{\prime}, I^{\prime}$ for both eigenvalues of $J$ to 'lie' inside the unit circle of the complex plane is expressed by the set of inequalities:

$$
1-\operatorname{Tr}(J)+\operatorname{Det}(J)>0, \quad 1+\operatorname{Tr}(J)+\operatorname{Det}(J)>0, \quad 1-\operatorname{Det}(J)>0,
$$

where

$$
\operatorname{Tr}(J)=2-\delta+\alpha\left(D^{\prime}-I^{\prime}\right), \quad \operatorname{Det}(J)=(1-\delta)\left[1+\alpha\left(D^{\prime}-I^{\prime}\right)\right]+\alpha \lambda I^{\prime}
$$

\footnotetext{
${ }^{5}$ This is in fact what happens with the linear case used in our examples (see Section 3).

${ }^{6}$ In this case, we use the ' symbol to denote, as usual, the first derivative.
} 
are the trace and the determinant of $J$, respectively. As is well known (see, e.g. Medio and Lines, 2001), condition (11) implies that the steady state is LAS, and the associated inequalities can be rewritten in terms of the parameters, respectively, as follows:

$$
\begin{gathered}
\delta\left(I^{\prime}-D^{\prime}\right)+\lambda I^{\prime}>0 \\
(2-\delta)\left[2+\alpha\left(D^{\prime}-I^{\prime}\right)\right]+\alpha \lambda I^{\prime}>0 \\
\delta+(1-\delta) \alpha\left(I^{\prime}-D^{\prime}\right)>\alpha \lambda I^{\prime} .
\end{gathered}
$$

Note first that (13) is certainly satisfied under our assumptions. As mentioned earlier, here we are not interested in analyzing conditions (13)-(15) in detail. Rather, we state a condition which is largely sufficient for the above inequalities to hold simultaneously. Very intuitively, this condition requires that neither the sum $\left|D^{\prime}\right|+I^{\prime}$ of the (absolute) slopes of demand and supply functions nor fraction $\lambda$ of the existing housing stock that contributes to the supply flow are too large, namely:

$$
\lambda<\delta+(1-\delta) \alpha\left(\left|D^{\prime}\right|+I^{\prime}\right)<1 .
$$

In fact, the right inequality in (16) is equivalent to $\left|D^{\prime}\right|+I^{\prime}<1 / \alpha$, or $1+\alpha\left(D^{\prime}-I^{\prime}\right)>0$, which implies (14). It also implies that $I^{\prime}<1 / \alpha$ and thus $\lambda>\alpha \lambda I^{\prime}$. Therefore, the left inequality in (16) implies (15). The parameter region defined by (16) is therefore strictly included in the parameter region in which the FSS is LAS. In order to focus merely on the effect of speculative behavior, in the following analysis we will select the parameters such that (16) holds and the underlying dynamical system without speculation is stable.

\subsection{General impact of speculative demand on housing prices}

We now consider the Jacobian matrix $J_{L}$ (of dimension $n:=L+2$ ) of the general dynamical system (5) with $L$ time lags in the speculative demand function, $L \geq 0$. In order to simplify the notation, we set

$$
\Phi_{0}^{\prime}=\Phi^{\prime}:=\frac{\partial \Phi}{\partial p}, \quad \Phi_{k}^{\prime}:=\frac{\partial \Phi}{\partial p_{-k}} \quad(k=1,2, \ldots, L),
$$

where the above derivatives are computed at the FSS. For $L \geq 1$ the general structure of the Jacobian matrix $J_{L}$ is sketched in (17), where the variables corresponding to each each row and column are also indicated:

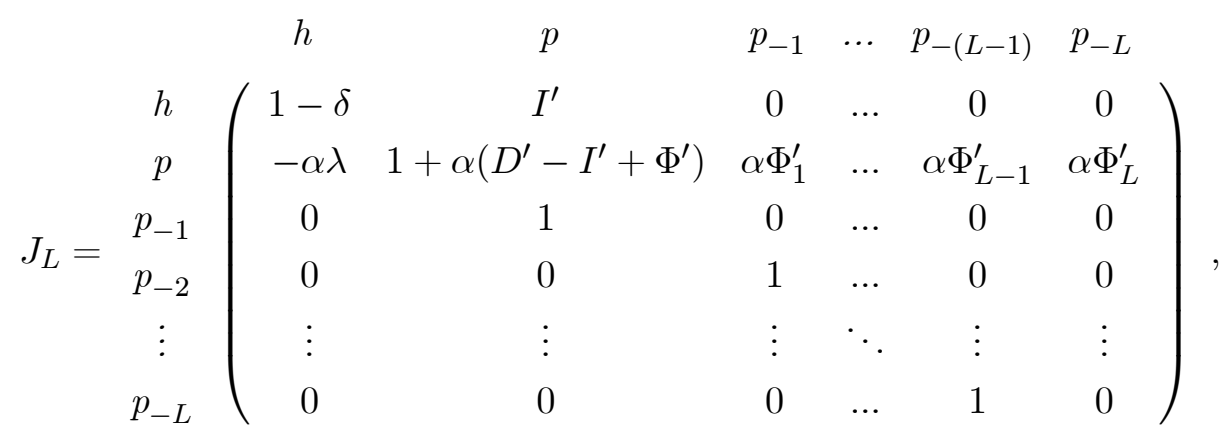


whereas for $L=0$ (the case in which speculative demand depends only on current price $p$ ) matrix $J_{0}$ is two-dimensional and its characteristic polynomial is determined as follows:

$$
\mathcal{P}_{0}(z)=\operatorname{det}\left(\begin{array}{cc}
1-\delta-z & I^{\prime} \\
-\alpha \lambda & 1+\alpha\left(D^{\prime}-I^{\prime}+\Phi^{\prime}\right)-z
\end{array}\right)=z^{2}-\operatorname{Tr}\left(J_{0}\right) z+\operatorname{Det}\left(J_{0}\right),
$$

where trace $\operatorname{Tr}\left(J_{0}\right)$ and determinant $\operatorname{Det}\left(J_{0}\right)$ are given as

$$
\operatorname{Tr}\left(J_{0}\right)=2-\delta+\alpha\left(D^{\prime}-I^{\prime}+\Phi^{\prime}\right), \quad \operatorname{Det}\left(J_{0}\right)=(1-\delta)\left[1+\alpha\left(D^{\prime}-I^{\prime}+\Phi^{\prime}\right)\right]+\alpha \lambda I^{\prime}
$$

Moreover, note that by eliminating the last column and row from (17) we obtain the Jacobian matrix $J_{L-1}$ of the case with $L-1$ lags. It follows that the characteristic polynomial of the general $L$-lag model, $\mathcal{P}_{L}(z)$, can be given a recursive representation in terms of $\mathcal{P}_{L-1}(z)$, the polynomial in the case of $L-1$ lags: ${ }^{7}$

$$
\mathcal{P}_{L}(z)=(-1)^{L} \alpha(1-\delta-z) \Phi_{L}^{\prime}-z \mathcal{P}_{L-1}(z)
$$

Let us now turn to some analytical results on stability. Generally speaking, the stability of the FSS depends on whether or not all the $n=L+2$ (real or complex) eigenvalues of (17) are of modulus smaller than unity. Denoting the eigenvalues of an $n \times n$ matrix $A$ by $z_{s}, s=1,2, \ldots, n$, it is also well known that, in general:

$$
\operatorname{Tr}(A)=\sum_{s=1}^{n} z_{s}, \quad \operatorname{Det}(A)=\prod_{s=1}^{n} z_{s} .
$$

Given the particular structure of matrix $J_{L}$ in (17), we can immediately write, for $L \geq 1$ :

$$
\operatorname{Tr}\left(J_{L}\right)=2-\delta+\alpha\left(D^{\prime}-I^{\prime}+\Phi^{\prime}\right)=\operatorname{Tr}\left(J_{0}\right), \quad \operatorname{Det}\left(J_{L}\right)=(-1)^{L} \alpha(1-\delta) \Phi_{L}^{\prime}
$$

If $\left|\Phi_{k}^{\prime}\right|$ is sufficiently small for any $k=0,1,2, \ldots, L$, the Jacobian matrix (17) is sufficiently 'close' to matrix $J$ given in (10) for the basic model without speculative demand. In this situation of 'weak' speculation, and under the assumed stability condition (16) for the 'real' economy, the FSS of the general system (5) with speculative demand is LAS as well. This follows immediately by continuity arguments, given that the eigenvalues of any matrix are continuous functions of the matrix coefficients (see, e.g. Stewart 2001, p. 37). Conversely, one can derive sufficient conditions on the partial derivatives $\Phi_{k}^{\prime}, k=0,1,2, \ldots, L$, under which speculative demand destabilizes an otherwise stable FSS. Based on (20) and on well-known properties of the modulus of complex numbers, we can write

$$
\left|\operatorname{Det}\left(J_{L}\right)\right|=\left|\prod_{s=1}^{n} z_{s}\right|=\prod_{s=1}^{n}\left|z_{s}\right|, \quad\left|\operatorname{Tr}\left(J_{L}\right)\right|=\left|\sum_{s=1}^{n} z_{s}\right| \leq \sum_{s=1}^{n}\left|z_{s}\right| .
$$

\footnotetext{
${ }^{7}$ This fact will prove useful in the four-dimensional model studied in Section 3.3.
} 
Assume now that none of the eigenvalues of $J_{L}$ are larger than unity in modulus, namely, $\left|z_{s}\right| \leq 1$, $s=1,2, \ldots, n=L+2$. Then it follows immediately that $\left|\operatorname{Det}\left(J_{L}\right)\right| \leq 1$ and $\left|\operatorname{Tr}\left(J_{L}\right)\right| \leq L+2$. Conversely, if

$$
\left|\operatorname{Det}\left(J_{L}\right)\right|>1 \quad \text { or } \quad\left|\operatorname{Tr}\left(J_{L}\right)\right|>L+2
$$

then at least one of the eigenvalues has modulus strictly greater than one, and the FSS is unstable. Condition (22) is therefore sufficient for the FSS to be unstable. It is clear that $\left|\operatorname{Det}\left(J_{L}\right)\right|, L \geq 1$, is a linearly increasing function of $\left|\Phi_{L}^{\prime}\right|$, whereas $\left|\operatorname{Det}\left(J_{0}\right)\right|$ and $\left|\operatorname{Tr}\left(J_{L}\right)\right|, L \geq 0$, increase linearly with $\left|\Phi^{\prime}\right|$ at least for sufficiently large $\left|\Phi^{\prime}\right| .^{8}$ In particular, for $L \geq 1$ and assuming ${ }^{9} \Phi^{\prime}>0$, the sufficient condition (22) corresponds to

$$
\left|\Phi_{L}^{\prime}\right|>\frac{1}{\alpha(1-\delta)} \quad \text { or } \quad \Phi^{\prime}>\left|D^{\prime}\right|+I^{\prime}+\frac{L+\delta}{\alpha} .
$$

Therefore, starting from a situation of stability of the FSS $\left(\left|\Phi_{k}^{\prime}\right|\right.$ small enough for any $k=$ $0,1,2, \ldots, L)$ and letting $\left|\Phi^{\prime}\right|$ or $\left|\Phi_{L}^{\prime}\right|$ increase beyond certain thresholds, the FSS switches from stable to unstable. In general, the derivatives $\Phi_{k}^{\prime}$ will depend on common parameters (for instance, a trend extrapolation parameter), and changes in these parameters will cause such derivatives to grow in modulus. Note also that conditions (22), or (23), are presented here merely to provide a general proof that speculation eventually destabilizes the FSS in this class of housing market models, but such conditions are in fact unnecessarily demanding. As is confirmed by the analytical and numerical investigation of the following particular models, various types of bifurcations occur at lower thresholds of the derivatives $\left|\Phi_{k}^{\prime}\right|$ than stated above.

The following analysis focuses on three 'popular' specifications of the impact of speculative demand, resulting in a 2-D, a 3-D and a 4-D model, respectively. As we shall see, the analysis of the (3-D) Jacobian matrix (17) in the case $L=1$ will be extremely useful in any such case. ${ }^{10}$ Therefore, in order to simplify and speed up the analysis of the following particular cases, we derive here a necessary and sufficient condition for all eigenvalues of $J_{L}, L=1$, to be inside the unit disk of the complex plane. As shown by Farebrother (1973), the roots of the third-degree polynomial:

$$
\mathcal{Q}(z)=z^{3}+a_{1} z^{2}+a_{2} z+a_{3}
$$

are all less than one in modulus iff the coefficients $a_{i}, i=1,2,3$, satisfy the set of inequalities:

$$
1+a_{1}+a_{2}+a_{3}>0, \quad 1-a_{1}+a_{2}-a_{3}>0, \quad 1-a_{2}+a_{3}\left(a_{1}-a_{3}\right)>0, \quad a_{2}<3 .
$$

Note that for $L=1$, the characteristic polynomial of $(17)$ is given by $\mathcal{P}_{1}(z):=\operatorname{det}\left(J_{1}-z \mathbf{I}\right)=-\mathcal{Q}(z)$,

\footnotetext{
${ }^{8}$ More precisely, if $\Phi^{\prime}$ is negative and increases in modulus, under our restrictions (16), $\left|\operatorname{Det}\left(J_{0}\right)\right|$ and $\left|\operatorname{Tr}\left(J_{L}\right)\right|$ increase with $\left|\Phi^{\prime}\right|$ only from certain thresholds onwards. We will not consider this situation in the forthcoming examples, since it is generally associated with strong (and unrealistic) overreaction by fundamental traders.

${ }^{9}$ This represents the most typical case in which destabilization occurs due to extrapolative demand from speculators who bet on the persistence of bull or bear markets, as shown in the forthcoming examples.

${ }^{10}$ It will even be useful in the four-dimensional model presented in Section 3.3.
} 
where the coefficients $a_{1}, a_{2}, a_{3}$ are defined as

$$
\begin{gathered}
a_{1}=-\left[2-\delta+\alpha\left(D^{\prime}-I^{\prime}+\Phi^{\prime}\right)\right]=-\operatorname{Tr}\left(J_{1}\right), \\
a_{2}=(1-\delta)\left[1+\alpha\left(D^{\prime}-I^{\prime}+\Phi^{\prime}\right)\right]+\alpha \lambda I^{\prime}-\alpha \Phi_{1}^{\prime}, \\
a_{3}=(1-\delta) \alpha \Phi_{1}^{\prime}=-\operatorname{Det}\left(J_{1}\right)
\end{gathered}
$$

Conditions (25) can thus be rewritten, respectively, as:

$$
\begin{gathered}
-\delta\left(D^{\prime}-I^{\prime}+\Phi^{\prime}+\Phi_{1}^{\prime}\right)+\lambda I^{\prime}>0 \\
(2-\delta)\left[2+\alpha\left(D^{\prime}-I^{\prime}+\Phi^{\prime}-\Phi_{1}^{\prime}\right)\right]+\alpha \lambda I^{\prime}>0, \\
(1-\delta)\left[1+\alpha\left(D^{\prime}-I^{\prime}+\Phi^{\prime}\right)\right]\left(1+\alpha \Phi_{1}^{\prime}\right)+\alpha \lambda I^{\prime}+\alpha \Phi_{1}^{\prime}(1-\delta)^{2}\left(1+\alpha \Phi_{1}^{\prime}\right)<1+\alpha \Phi_{1}^{\prime}, \\
(1-\delta)\left[1+\alpha\left(D^{\prime}-I^{\prime}+\Phi^{\prime}\right)\right]+\alpha \lambda I^{\prime}<3+\alpha \Phi_{1}^{\prime} .
\end{gathered}
$$

In particular, assume an initial situation in which such inequalities hold simultaneously, and therefore the FSS is stable. If $\Phi^{\prime}$ or $\Phi_{1}^{\prime}$ vary (possibly depending on the variation of a common parameter) such that (only) the first condition in (26) is violated, then one of the three eigenvalues becomes larger than 1 . This might be associated, in general, with a saddle-node, a pitchfork, or a transcritical bifurcation. Violation of the second inequality is associated with a flip bifurcation (one eigenvalue becomes smaller than -1 ), whereas violation of the third inequality is associated with a Neimark-Sacker bifurcation (two complex conjugate eigenvalues become larger than one in modulus). The next sections illustrate these general findings.

\section{The impact of speculative demand in three significant cases}

For simplicity, speculative demand $\Phi$ is modeled in such a way that $\Phi\left(p^{*}, p^{*}, p^{*}, \ldots, p^{*}\right)=0$, so that it vanishes when the system evolves along the steady state solution path. This assumption is satisfied by the most common specifications of speculative trading rules within the heterogeneous agent literature (this is true, in particular, for trend-following rules and fundamental-based rules). Note that speculative demand can be positive or negative in our model, and therefore the speculative component $\Phi$ is simply interpreted as a positive or negative correction to the real demand $D$. Alternatively, a negative speculative component can be interpreted as an additional (positive) amount of housing for sale in the period. We explore the dynamics under three different specifications of $\Phi$. Each specification captures in a different way the interplay between heterogeneous investment rules (an extrapolative or trend-following rule and a fundamental-based rule) with possible endogenous mechanisms of switching between different types of behavior.

In the numerical investigation and graphical examples we will use a linear specification of demand and supply functions, namely:

$$
D(p)=\beta_{0}-\beta p,
$$




$$
I(p)=-\theta_{0}+\theta p
$$

where $\beta_{0}, \beta, \theta_{0}, \theta$, are strictly positive parameters. ${ }^{11}$ It follows that the FSS defined by (7) is specified as

$$
h^{*}=\frac{\theta \beta_{0}-\theta_{0} \beta}{\delta \beta+(\lambda+\delta) \theta}, \quad p^{*}=\frac{\delta \beta_{0}+(\lambda+\delta) \theta_{0}}{\delta \beta+(\lambda+\delta) \theta},
$$

provided that $\theta \beta_{0}-\theta_{0} \beta>0$ or, equivalently, $p^{*}>p_{\min }:=\theta_{0} / \theta$. It also follows that $D^{\prime}=-\beta$, $I^{\prime}=\theta$.

With regard to the 'real' parameters common to all the models presented in the following subsections, our base parameter selection is as follows: $\alpha=0.5, \delta=0.02, \lambda=0.02$. Moreover, we assume that the real demand curve has (absolute) slope $\beta=0.05$, whereas the slope of the curve representing new housing investment, $\theta$, will possibly vary across examples. A few comments are in order on our choice of parameters describing the 'real' economy. Assuming that the time unit is one year, a depreciation rate $\delta=0.02$ implies a realistic half-life of a housing unit of roughly 35 years, whereas $\lambda=0.02$ means that $2 \%$ of homeowners per year sell their houses, which seems all in all reasonable. In contrast, there is no specific rationale behind the values assigned to the remaining parameters (the price adjustment coefficient $\alpha$ and the slopes of the real demand and supply curves $\beta$ and $\theta$ ); they serve only illustrative purposes. In fact, given that the model has linear (real) demand and supply and is expressed in absolute changes of housing price and stock, a suitable calibration of such parameters would require the preliminary specification of the price level and of the unit of measure of the stock of housing. On the other hand, both the price level and the specification of the 'housing unit' are rather uninfluent to the results presented in the sequel, in that the model can be reformulated in deviations from the FSS under our assumptions (see later on in this section). ${ }^{12}$ Concerning the parameters characterizing speculative behavior, the extrapolative demand coefficient will usually be regarded as the bifurcation parameter. In general, we will run experiments under two alternative scenarios for the 'supply response' parameter $\theta$, namely, a situation where the supply curve of new housing is sufficiently flat (low $\theta$ ), and a situation where it is more sloped (large $\theta$ ). Note, however, that in order to simplify our analysis and to get a clearer picture of the results, changes in the slope $\theta$ will always be accompanied by suitable adjustments of the intercept $\theta_{0}$, such that the steady state coordinates remain unaffected by such parameter changes. Similar adjustments could be performed for the parameters $\beta$ and $\beta_{0}$ of the demand curve, as well. In fact, with a simple change of coordinates in the parameter space, the model can be rewritten in terms of new parameters $h^{*}$ and $p^{*}$ (replacing $\beta_{0}$ and $\theta_{0}$ ), that can thus be interpreted

\footnotetext{
${ }^{11}$ Note in particular that the supply function (28) can be obtained from a standard profit maximization setup with a quadratic cost function. Consistent with this setup, the optimal amount $I(p)$ of new constructions is positive iff $p>\theta_{0} / \theta:=p_{\min }$. Taking into account this constraint properly would result in a piecewise-smooth dynamical system. Similar natural constraints involving upper and lower bounds on the variables may even result in piecewise-continuous systems. We remark here that Laura largely contributed in recent years to developing completely new analytical and numerical tools to deal with these kinds of maps (more details are provided in the concluding section). We hope to be able to 'exploit' Laura's great experience in this field and to collaborate with her in the future on a possible extension of this work. As for now, we implicitly assume in our numerical experiments that fixed parameter $\theta_{0}$ is such that price $p$ never falls below the above threshold.

${ }^{12}$ Parameter calibration would, of course, be important in the case of isoelastic demand and supply and if the laws of motion were specified in relative price and stock adjustments.
} 
as the (exogenous) coordinates of the FSS. ${ }^{13}$ Without loss of generality, our numerical experiments will thus focus on the deviations $\left(h-h^{*}\right)$ and $\left(p-p^{*}\right)$ of housing stock and prices from their FSS levels, respectively.

\subsection{The dynamic interplay between extrapolative and regressive demand}

This specification, which dates back to Day and Huang (1990), has often been adopted in the heterogeneous agent literature, and represents the most parsimonious way to capture the interplay between extrapolative and regressive beliefs (see, e.g. De Grauwe et al. 1993, Dieci and Westerhoff 2010). Extrapolators believe that house prices include a (positive or negative) bubble component that will continue to grow geometrically. Therefore, their excess demand is proportional, in absolute terms, to the current deviation from the fundamental price, $\left|p_{t}-p^{*}\right|$, with the sign of $\left(p_{t}-p^{*}\right)$. In contrast, agents relying on regressive beliefs, or fundamentalists, believe that the existing deviation from the fundamental price will partly collapse in the next period and therefore their excess demand, again proportional to $\left|p_{t}-p^{*}\right|$ in absolute terms, has the sign of $\left(p^{*}-p_{t}\right)$. We denote by $w_{t}$ the market fraction of extrapolators in period $t$. Proportion $w_{t}$ is assumed to change endogenously as a function of market circumstances. In particular, the more the price deviates from its fundamental value, the more speculators will switch from extrapolative expectations to regressive expectations, as they fear that the bubble will burst soon. Therefore we have

$$
w_{t}=\frac{\omega}{1+\nu\left(p_{t}-p^{*}\right)^{2}}
$$

where the parameter $\omega, 0 \leq \omega \leq 1$, represents the maximum fraction of agents using the extrapolative rule, and $\nu>0$ is a sensitivity parameter. Clearly, the larger $\nu$, the faster speculators switch from extrapolative behavior to mean reverting behavior, as the bubble becomes more extreme. Extrapolative demand and regressive demand in period $t$ are thus expressed as $w_{t} \gamma\left(p_{t}-p^{*}\right)$ and $\left(1-w_{t}\right) \psi\left(p^{*}-p_{t}\right)$, respectively, where $\gamma, \psi>0$. Speculative demand $\Phi$ is the sum of such components and depends therefore only on the current price (such that $L=0$ ), as follows:

$$
\Phi\left(p_{t}\right)=\psi\left(p^{*}-p_{t}\right)+w_{t}(\gamma+\psi)\left(p_{t}-p^{*}\right)
$$

The dynamical system in the presence of speculative demand with $L=0$ thus remains twodimensional, as the base model without speculation, and is represented by the nonlinear map:

$$
\left\{\begin{array}{l}
h^{\prime}=(1-\delta) h+I(p) \\
p^{\prime}=p+\alpha(D(p)+\Phi(p)-I(p)-\lambda h)
\end{array},\right.
$$

where

$$
\Phi(p)=\psi\left(p^{*}-p\right)+\frac{\omega}{1+\nu\left(p-p^{*}\right)^{2}}(\gamma+\psi)\left(p-p^{*}\right),
$$

\footnotetext{
${ }^{13}$ In particular, the model can then be rewritten in deviations from the FSS, via the change of variables $\eta:=h-h^{*}$, $\pi:=p-p^{*}$. The model in deviations with linear demand and supply is independent of parameters $h^{*}$ and $p^{*}$ (or $\beta_{0}$ and $\left.\theta_{0}\right)$, as can be checked.
} 
while $D, I$, are specified according to (27) and (28), respectively. It follows that

$$
\Phi^{\prime}:=\frac{d \Phi}{d p}\left(p^{*}\right)=-\psi+\omega(\gamma+\psi)=\omega \gamma-(1-\omega) \psi
$$

and $\Phi_{k}^{\prime}=0$ for $k \geq 1$. Using conditions (26) with $\Phi_{1}^{\prime}=0$, the region of local asymptotic stability of the FSS turns out to be defined by the set of inequalities: ${ }^{14}$

$$
\begin{gathered}
\delta[\beta+\theta-\omega \gamma+(1-\omega) \psi]+\lambda \theta>0, \\
(2-\delta)[2+\alpha(\omega \gamma-(1-\omega) \psi-(\beta+\theta))]+\alpha \lambda \theta>0, \\
(1-\delta)[1+\alpha(\omega \gamma-(1-\omega) \psi-(\beta+\theta))]+\alpha \lambda \theta<1 .
\end{gathered}
$$

Note that the second condition in (33) is satisfied, provided that the regressive demand parameter $\psi$ is not too large. ${ }^{15}$ Under this assumption, and focusing on the behavior of the extrapolative demand parameter $\gamma$, the first and third inequalities can be written, respectively, as:

$$
\begin{gathered}
\gamma<\frac{1}{\omega}\left[\beta+(1-\omega) \psi+\frac{\lambda+\delta}{\delta} \theta\right]:=\gamma_{P}, \\
\gamma<\frac{1}{\alpha \omega}\left[\frac{\delta-\alpha \lambda \theta}{1-\delta}+\alpha(\beta+\theta+(1-\omega) \psi)\right]:=\gamma_{N S} .
\end{gathered}
$$

Note first that bifurcation values $\gamma_{P}$ and $\gamma_{N S}$ are strictly positive under our parameter restrictions. This implies that conditions (34) and (35) are satisfied when $\gamma=0$. Therefore, by increasing the extrapolation parameter $\gamma$ starting from $\gamma=0$, a different type of bifurcation will take place, depending on whether $\gamma_{P}$ is smaller or larger than $\gamma_{N S}$. Generally speaking, $\gamma_{P}<\gamma_{N S}$ if parameter $\theta$ is small enough, whereas the opposite is true for larger $\theta$. Therefore, the slope of the housing supply curve turns out to be crucial as to what kind of local bifurcation occurs to the FSS when extrapolation becomes stronger. ${ }^{16}$ In the first case, a pitchfork bifurcation takes place, at which one of the eigenvalues of the Jacobian matrix becomes equal to 1, the FSS becomes unstable and two new stable steady states are created, in symmetric positions, around the unstable FSS. The latter result can easily be checked by looking at possible additional steady states of (32), which requires solving a simple cubic equation: it turns out that, in general, only one FSS exists for $0 \leq \gamma<\gamma_{P}$, whereas three steady states exist for $\gamma>\gamma_{P}$. In the second case, a (supercritical) Neimark-Sacker bifurcation takes place, at which the modulus of the complex conjugate eigenvalues becomes equal to and then larger than one, and an invariant attracting closed curve is created around the unstable FSS. Figures 1 and 2 report the results of some numerical experiments on the impact of $\gamma$, for $\omega=1, \psi=5$ and $\nu=0.01$. Housing price and stock are represented in

\footnotetext{
${ }^{14}$ Equivalently, these inequalities can be directly derived from the 2-D Jacobian matrix of system (32).

${ }^{15}$ In particular, this condition is always satisfied (under parameter restriction (16)) if $\omega=1$, i.e. if no exogenous upper bound is imposed on the market impact of extrapolators, because in this case $\Phi^{\prime}=\gamma$ does not depend on parameter $\psi$.

${ }^{16} \mathrm{On}$ the contrary, it turns out from the comparison of (34) and (35) that the (absolute) slope $\beta$ of the 'real' demand curve has no specific influence on the type of bifurcation occurring when $\gamma$ increases.
} 
deviations from the FSS. ${ }^{17}$ For $\theta=0.025$ (supply curve of new construction is relatively flat), the bifurcation diagram in Figure 1 (top panel) represents the asymptotic behavior of the housing price for increasing values of parameter $\gamma$, assuming the initial price is slightly above the fundamental price. Note that a symmetric plot can be obtained by taking the initial price slightly below $p^{*}$. Since in this case $\gamma_{P}=0.1<\gamma_{N S} \cong 0.1153$, the loss of stability takes place via a pitchfork bifurcation, creating two new coexisting fixed points characterized by a higher and a lower price and housing stock, respectively, than the FSS. Each of the two coexisting non-fundamental steady states then undergoes a sequence of period doubling bifurcations, resulting in cyclical and eventually chaotic price dynamics, restricted to either the 'bull' market (high price and housing stock) or the 'bear' market (low price and housing stock). When the parameter $\gamma$ becomes larger than a certain threshold, housing prices tend to switch endogenously between bull and bear market regions. The behavior of price and housing stock versus time is represented, for $\gamma=7.3$, in the middle and bottom panels.

\section{*** FIGURE 1 APPROXIMATELY HERE ***}

Figure 2 is obtained for a much larger value of $\theta$, namely $\theta=0.5$. In this case, $\gamma_{N S} \cong$ $0.5806<\gamma_{P}=1.05$, and therefore the local bifurcation causing the loss of stability of the FSS is a Neimark-Sacker bifurcation. However, as the bifurcation diagram suggests (top panel), for some range of parameter $\gamma\left(\operatorname{such}\right.$ that $\left.\gamma>\max \left(\gamma_{P}, \gamma_{N S}\right)\right)$ the stable invariant closed curve generated by the Neimark-Sacker bifurcation of the FSS coexists with two locally stable non-fundamental steady states, and surrounds all the steady states and their basins of attraction. This coexistence scenario, which we do not explore in detail, occurs quite frequently in models in which a 'normal' steady state may become unstable via both a pitchfork and a Neimark-Sacker bifurcation, and generically entails very complicated dynamics and 'contact' bifurcations involving attracting and repelling invariant closed curves, and stable manifolds of saddle cycles (see, e.g. Agliari et al. 2007). Finally note that, whatever the local bifurcation causing the loss of stability of the FSS, the scenario that prevails for very large values of the coefficient $\gamma$ is a 'pitchfork scenario' followed by a regime where switches between phases of high and low prices occur at seemingly unpredictable points in time (middle panel). The role of fundamentalist demand parameter $\psi$ is not neutral to such scenarios. Here we have chosen a relatively large value of $\psi$. The combination of large values ${ }^{18}$ of $\gamma$ and $\psi$ turns out to be essential for such a chaotic regime to occur. Quite differently, choosing a much smaller value of $\psi$ $(\psi=0.5)$, all other parameters being unchanged, leads to a scenario in which the non-fundamental steady states remain locally stable and never bifurcate further, no matter how large the parameter $\gamma$ is (the only effect of the parameter $\gamma$ is to increase their deviation from the FSS). Finally, let us now briefly summarize the role played by the 'supply response' parameter, $\theta$. A prompt response (large $\theta$ ), combined with sufficiently large strength $\gamma$ of extrapolative demand, causes the FSS to lose stability via a Neimark-Sacker bifurcation: this kind of quasiperiodic behavior implies that

\footnotetext{
${ }^{17}$ Recall that parameters $\beta_{0}$ and $\theta_{0}$ (or, alternatively, $h^{*}$ and $p^{*}$ ) can be arbitrarily chosen without affecting the numerical results presented below.

${ }^{18}$ See Section 3.3 for a brief discussion of the relationship between demand parameters and price expectations of the two types of agents.
} 
the growth of the bubble is followed by an endogenous crash. On the contrary, a slow response of the supply of new constructions to increasing demand and increasing prices (small $\theta$ ) leads to a scenario in which the economy tends to remain stuck in a non-fundamental steady state. Moreover, if $\theta$ is small, even when the system is unstable and intricate switches between bull and bear markets occur for very large $\gamma$, housing stock changes slowly and smoothly compared to prices. However, changing the supply parameter from $\theta=0.025$ to $\theta=0.5$ affects the amplitude of stock fluctuations remarkably, in face of identical ranges of price fluctuations (compare the middle and bottom panels in Figure 1 with the corresponding panels in Figure 2)

\section{*** FIGURE 2 APPROXIMATELY HERE ***}

\subsection{Interaction of fundamentalists and trend-followers with fixed proportions}

In this case, chartists are modeled as true trend extrapolators. In other words, unlike the previous specification, here chartists do not rely on estimates of the 'fundamental' and the 'bubble' components of housing prices, and of the likely future development of the latter. Their speculative demand is represented as an increasing function of a trend signal. Here we simply assume that the trend signal is the most recent price movement ${ }^{19}$, and we use an increasing $S$-shaped demand function for chartist speculative demand. The proportions of investors using trend-following and fundamentalbased rules are fixed, and $w_{t}=\bar{w}$ denotes the market proportion of chartists. Speculative demand is thus given by:

$$
\begin{aligned}
\Phi\left(p_{t}, p_{t-1}\right) & =\bar{w} \kappa \tanh \left(\frac{\mu}{\kappa}\left(p_{t}-p_{t-1}\right)\right)+(1-\bar{w}) \psi\left(p^{*}-p_{t}\right)= \\
& \psi\left(p^{*}-p_{t}\right)+\bar{w}\left[\kappa \tanh \left(\frac{\mu}{\kappa}\left(p_{t}-p_{t-1}\right)\right)-\psi\left(p^{*}-p_{t}\right)\right],
\end{aligned}
$$

where $\mu, \kappa, \psi>0$.

The nonlinearity introduced via the hyperbolic tangent function can be justified in terms of chartist risk perception in the presence of very large price movements (Chiarella et al. 2002, Chiarella et al. 2006). ${ }^{20}$ The nonlinear dynamical system with fundamentalists, trend-followers and fixed market impact becomes three-dimensional $(L=1)$. It is specified through the map:

$$
\left\{\begin{array}{l}
h^{\prime}=(1-\delta) h+I(p) \\
p^{\prime}=p+\alpha\left(D(p)+\Phi\left(p, p_{-1}\right)-I(p)-\lambda h\right) \\
p_{-1}^{\prime}=p
\end{array}\right.
$$

\footnotetext{
${ }^{19}$ More generally, the trend signal may be modeled as the deviation of the latest observation from a time average computed over the last $N$ periods, or even as the deviation between short-term and long-term moving averages. However, these more realistic specifications would increase the dimension of the dynamical system considerably. See, e.g. Chiarella et al. (2006).

${ }^{20}$ In fact, the chartist demand component in function (36) can again be written as $\bar{w} \mu_{t}\left(p_{t}-p_{t-1}\right)$, where the trend extrapolation coefficient $\mu_{t}$ is now state-dependent and attains its maximum, $\mu$, when the trend signal $\left|p_{t}-p_{t-1}\right| \rightarrow 0$, whereas $\mu_{t}$ decreases as $\left|p_{t}-p_{t-1}\right|$ becomes larger. Unlike a linear function with constant slope $\mu$, this demand function thus partly 'levels off' if larger price movements are observed.
} 
where

$$
\Phi\left(p, p_{-1}\right)=\psi\left(p^{*}-p\right)+\bar{w}\left[\kappa \tanh \left(\frac{\mu}{\kappa}\left(p-p_{-1}\right)\right)-\psi\left(p^{*}-p\right)\right] .
$$

It follows that

$$
\Phi^{\prime}:=\frac{\partial \Phi}{\partial p}\left(p^{*}, p^{*}\right)=-\psi+\bar{w}(\mu+\psi)=\bar{w} \mu-(1-\bar{w}) \psi, \quad \Phi_{1}^{\prime}:=\frac{\partial \Phi}{\partial p_{-1}}\left(p^{*}, p^{*}\right)=-\bar{w} \mu
$$

and $\Phi_{k}^{\prime}=0$ for $k \geq 2$. Using conditions (26), the region of local asymptotic stability of the FSS is defined by the set of inequalities:

$$
\begin{gathered}
\delta[\beta+\theta+(1-\bar{w}) \psi]+\lambda \theta>0, \\
(2-\delta)[2+\alpha(2 \bar{w} \mu-(1-\bar{w}) \psi-(\beta+\theta))]+\alpha \lambda \theta>0, \\
(1-\delta)[1+\alpha(\bar{w} \mu-(1-\bar{w}) \psi-(\beta+\theta))](1-\alpha \bar{w} \mu)+\alpha \lambda \theta-(1-\delta)^{2} \alpha \bar{w} \mu(1-\alpha \bar{w} \mu)<1-\alpha \bar{w} \mu, \\
(1-\delta)[1+\alpha(\bar{w} \mu-(1-\bar{w}) \psi-(\beta+\theta))]+\alpha \lambda \theta<3-\alpha \bar{w} \mu .
\end{gathered}
$$

Unlike the previous case, here the first condition is always true under the assumed natural restrictions on the parameters. Put differently, the trend-following behavior of the chartists rules out the possibility of multiple steady states and pitchfork bifurcations. ${ }^{21}$ Similarly to the previous case, the second condition in (37) is satisfied if the regressive parameter $\psi$ is not too large, and we assume this is the case in what follows. Focusing on the impact of the extrapolation parameter $\mu$ in the range $V:=\left(0, \frac{1}{\alpha \bar{w}}\right)$, for fixed values of the remaining parameters, the third inequality is equivalent to:

$$
A(\mu)<B(\mu)+C
$$

where

$$
A(\mu):=(1-\delta)(1+\alpha \delta \bar{w} \mu), \quad B(\mu):=1-\frac{\alpha \lambda \theta}{1-\alpha \bar{w} \mu}, \quad C:=\alpha(1-\delta)(\beta+\theta+(1-\bar{w}) \psi) .
$$

Note that here parameter $\mu$ plays a somewhat similar role for steady state stability as does the parameter $\gamma$ in the model developed in the previous section. In particular, by setting $\mu=0$ in (37) and $\gamma=0$ in (33), we obtain two formally identical sets of conditions (with $\bar{w}$ replacing $\omega)$. Therefore, based on our discussion on the third inequality of (33) in the previous section, we conclude that $A(0)<B(0)+C$. While $A(\mu)$ increases linearly with $\mu, B(\mu)$ decreases monotonically from $B(0)>0$ to $-\infty$ as $\mu$ ranges in $V=\left(0, \frac{1}{\alpha \bar{w}}\right)$. Therefore, provided that the fourth inequality in (37) is satisfied for any $\mu \in V$, a Neimark-Sacker bifurcation must occur at some value $\mu_{N S}$ in that interval. ${ }^{22}$ In Figure 3 we assume $\psi=5, \bar{w}=0.5, \kappa=100$. With these parameters, the fourth inequality in (37) is satisfied for any $\theta>0$ and any $\mu \in V$. Assuming further $\theta=0.5$, a supercritical

\footnotetext{
${ }^{21}$ Intuitively, at a non-fundamental steady state, fundamentalist demand would be different from zero, whereas trend-based chartist demand vanishes at any steady state solution. This situation of permanent excess demand would set in motion price corrections towards the FSS.

${ }^{22}$ If the parameter $\lambda$ is small, the bifurcation value $\mu_{N S}$ is indeed very close to the upper bound of the interval, $1 /(\alpha \bar{w})$.
} 
Neimark-Sacker bifurcation occurs at $\mu=\mu_{N S} \cong 3.9866<1 /(\alpha \bar{w})=4$, and the bifurcation diagram for the price (top-left panel) shows that the size of quasiperiodic oscillations increases with the trend-following parameter $\mu$. A closer look at the phase plane shows that the attracting invariant closed curve created through the Neimark-Sacker bifurcation undergoes a sequence of qualitative changes leading to more complex attractors (see, e.g. the top-right panel, where $\mu=$ 6.9). The mechanisms behind such changes are due to the non-invertibility of the map, and have been illustrated in great detail in related work on financial market dynamics with fundamentalists and chartists (Chiarella et al. 2002). Moreover, the top-left panel reveals that periodic motion prevails for very large values of $\mu$. We know from the foregoing local stability analysis that the parameter $\theta$ has no effect on the types of local bifurcation that may occur under the effect of increasing trend extrapolation. However, if the housing supply curve is much flatter, we detect remarkable changes in the global picture of the phase space. For $\theta=0.025$, the bottom-left (price) and right (stock) diagrams suggest that coexisting attractors emerge out of the invariant curve in this case, and therefore the combination of the initial condition and parameter $\mu$ becomes crucial for long-run evolution. Such coexisting periodic attractors again represent alternative possible scenarios of 'bull markets' and 'bear markets', in the sense that the (average) price and stock are higher (lower) than their fundamental levels.

\section{*** FIGURE 3 APPROXIMATELY HERE ***}

\subsection{Evolutionary switching between fundamental and trend-following rules}

The demand of fundamentalists and trend-followers is specified very similarly to the previous case (the only difference being that chartist demand here is represented by a linear function), but their proportions evolve endogenously according to a multinomial logit model, based on a certain measure of the rules' performance. This approach has been widely adopted in the literature on financial market modeling and macroeconomic modeling with heterogeneous agents. ${ }^{23}$ The 'fitness' measure that we adopt here is the (negative) squared prediction error relative to the most recent price forecast (see, e.g. Parke and Waters 2007, Lines and Westerhoff 2011). Generally speaking, we may assume that investors' speculative demand in each period is proportional to their expected unit profit in the period or, put differently, to the expected house price change. The expectations of price $p_{t+1}$ taken at the beginning of period $t$ by chartists and fundamentalists are modeled, respectively, as follows:

$$
p_{t+1}^{e, C}:=p_{t}+\widehat{\mu}\left(p_{t}-p_{t-1}\right), \quad p_{t+1}^{e, F}:=p_{t}+\widehat{\psi}\left(p^{*}-p_{t}\right)
$$

where $\widehat{\mu}, \widehat{\psi}>0$, so that the (unweighted) chartist and fundamentalist speculative demand components become

$$
D_{t}^{C}:=q^{C}\left(p_{t+1}^{e, C}-p_{t}\right)=\mu\left(p_{t}-p_{t-1}\right)
$$

\footnotetext{
${ }^{23}$ For applications to evolutionary finance see, e.g. Brock and Hommes (1998), Hommes (2001), Chiarella and He (2002), Westerhoff (2004), De Grauwe and Grimaldi (2006). Applications to (macro)economic dynamics include Brock and Hommes (1997), Lines and Westerhoff (2011) and De Grauwe (2010).
} 


$$
D_{t}^{F}:=q^{F}\left(p_{t+1}^{e, F}-p_{t}\right)=\psi\left(p^{*}-p_{t}\right)
$$

where $q^{C}, q^{F}>0, \mu:=q^{C} \widehat{\mu}>0, \psi:=q^{F} \widehat{\psi}>0 .{ }^{24}$ The speculative demand of each agent type is thus positive (negative) if an upward (downward) price movement is expected, and zero if no price change is expected. Note that price expectations at the beginning of a given period are based, in general, on the price history up to that date. Therefore, the forecast errors of trend-followers and fundamentalists relative to period $t-1$ (as computed once price $p_{t}$ is revealed) can be written, respectively, by ${ }^{25}$

$$
\begin{gathered}
p_{t}^{e, C}-p_{t}=p_{t-1}+\widehat{\mu}\left(p_{t-1}-p_{t-2}\right)-p_{t}, \\
p_{t}^{e, F}-p_{t}=p_{t-1}+\widehat{\psi}\left(p^{*}-p_{t-1}\right)-p_{t} .
\end{gathered}
$$

The performance measures of the trend-following and fundamental rules are thus given by

$$
A_{t}^{C}=-\left(p_{t}^{e, C}-p_{t}\right)^{2}, \quad A_{t}^{F}=-\left(p_{t}^{e, F}-p_{t}\right)^{2}-K
$$

Note that we have added a possible negative correction $-K, K \geq 0$, to the 'attractiveness' of the fundamentalist rule. This negative term may be justified as the cost needed to estimate the fundamental price and the speed of adjustment towards it, which requires a sufficiently deep knowledge of the working of the economy. Alternatively, this negative component may be justified in terms of a 'behavioral bias' of the fundamentalists. ${ }^{26}$ Finally, the market impact of trend-followers in period $t$ is given by:

$$
w_{t}=\frac{\exp \left(\tau A_{t}^{C}\right)}{\exp \left(\tau A_{t}^{C}\right)+\exp \left(\tau A_{t}^{F}\right)},
$$

where $A_{t}^{C}$ and $A_{t}^{F}$ are given by (42) and the parameter $\tau>0$ represents the so-called 'intensity of choice' (see, e.g. Brock and Hommes 1997).

The dynamical system with speculative demand generated by trend-followers and fundamentalists in this case becomes four-dimensional $(L=2)$, specified by the map:

$$
\left\{\begin{array}{l}
h^{\prime}=(1-\delta) h+I(p) \\
p^{\prime}=p+\alpha\left(D(p)+\Phi\left(p, p_{-1}, p_{-2}\right)-I(p)-\lambda h\right) \\
p_{-1}^{\prime}=p \\
p_{-2}^{\prime}=p_{-1}
\end{array},\right.
$$

where

$$
\Phi\left(p, p_{-1}, p_{-2}\right)=\psi\left(p^{*}-p\right)+\frac{\exp \left(\tau A^{C}\right)}{\exp \left(\tau A^{C}\right)+\exp \left(\tau A^{F}\right)}\left[\mu\left(p-p_{-1}\right)-\psi\left(p^{*}-p\right)\right]
$$

\footnotetext{
${ }^{24} \mathrm{~A}$ very similar interpretation of the speculative demand function in terms of expected unit profits applies also to the models studied in the previous sections.

${ }^{25}$ Note that the forecast errors in equations (40) and (41) can also be interpreted as the difference between the expected and the actual price change in period $t-1$. For instance, in the case of chartists: $p_{t}^{e, C}-p_{t}=\left(p_{t}^{e, C}-p_{t-1}\right)-$ $\left(p_{t}-p_{t-1}\right)=\widehat{\mu}\left(p_{t-1}-p_{t-2}\right)-\left(p_{t}-p_{t-1}\right)$.

${ }^{26}$ See, e.g. Lines and Westerhoff $(2011)$ for a discussion of this point within a macro-model with heterogeneous inflationary expectations.
} 
and where:

$$
\begin{gathered}
\left.A^{C}\left(p, p_{-1}, p_{-2}\right)=-\left[p_{-1}+\widehat{\mu}\left(p_{-1}-p_{-2}\right)-p\right)\right]^{2}, \\
A^{F}=A^{F}\left(p, p_{-1}\right)=-\left[p_{-1}+\widehat{\psi}\left(p^{*}-p_{-1}\right)-p\right]^{2}-K .
\end{gathered}
$$

Denoting by

$$
w^{*}:=\frac{1}{1+\exp (-\tau K)}
$$

the chartist proportion at the FSS, the partial derivatives of the speculative demand function (evaluated at $\left.\left(p^{*}, p^{*}, p^{*}\right)\right)$ turn out to be formally identical to the corresponding derivatives in the case of fixed fractions, namely:

$$
\Phi^{\prime}:=\frac{\partial \Phi}{\partial p}=w^{*} \mu-\left(1-w^{*}\right) \psi, \quad \Phi_{1}^{\prime}:=\frac{\partial \Phi}{\partial p_{-1}}=-w^{*} \mu, \quad \Phi_{2}^{\prime}:=\frac{\partial \Phi}{\partial p_{-2}}=0
$$

and $\Phi_{k}^{\prime}=0$ for $k \geq 3$. Although $\Phi$ is now a function of observed prices in three subsequent periods, its partial derivative with respect to the earliest price, $\Phi_{2}^{\prime}$, is zero at the FSS. This is due to the fact that the fitness of the rules depends on squared forecast errors, and therefore the terms in square brackets in (44)-(45) (which vanish at the FSS) appear as multiplicative factors in the partial derivatives of $A^{C}$ and $A^{F}$ with respect to prices. The latter are therefore all equal to zero at the FSS. Moreover, the chartist weight $w$ in (43) can be regarded as a function of current and past prices through $A^{C}$ and $A^{F}$, namely, $w=f\left(A^{C}, A^{F}\right)$, where $A^{C}$ and $A^{F}$ are given by (44) and (45). Therefore, at the FSS:

$$
\frac{\partial w}{\partial p}=\frac{\partial f}{\partial A^{C}} \frac{\partial A^{C}}{\partial p}+\frac{\partial f}{\partial A^{F}} \frac{\partial A^{F}}{\partial p}=0
$$

and similarly for $\partial w / \partial p_{-1}, \partial w / \partial p_{-2}$, which proves (46). Given that $\Phi_{2}^{\prime}=0$, and remembering the recurrent representation (19) of the characteristic polynomial, it follows that the four-dimensional Jacobian matrix in this case has one eigenvalue equal to zero, whereas the three remaining eigenvalues have moduli smaller than one iff conditions (37) hold (with $w^{*}$ replacing the exogenous proportion $\bar{w}) .{ }^{27}$ The same results of the previous model with fixed fractions regarding the loss of stability and the local bifurcation (due to strong trend-chasing behavior) apply to the present model, too. In particular, a Neimark-Sacker bifurcation occurs at some value $\mu_{N S}$ in interval $V=$ $\left(0, \frac{1}{\alpha w^{*}}\right) \cdot{ }^{28}$ Of course, apart from the linearized behavior around the FSS, the model with timevarying proportions of fundamentalists and trend-followers may produce quite different dynamics from the corresponding fixed fraction model. Assuming $\theta=0.5$, Figure 4 reports the results of numerical experiments carried out with parameters $\psi=0.4, q^{C}=q^{F}=1, \tau=0.003, K=0$. As the bifurcation diagram of the price against trend extrapolation parameter $\mu$ confirms (top panel), the loss of stability occurs again via a (supercritical) Neimark-Sacker bifurcation. Quasiperiodic motion alternates with 'windows' of periodic motion for larger values of $\mu$. Beyond a certain threshold for

\footnotetext{
${ }^{27}$ Note that the set of conditions (26) turns out to be extremely useful in all cases studied in the present paper.

${ }^{28}$ Again we assume that the second and fourth inequalities in (37) are satisfied for any $\mu \in V$, which is the case in the following numerical example.
} 
parameter $\mu$ (around $\mu=6.7$ in this example), there exists a parameter range such that the initial condition is crucial for the asymptotic dynamics, due to the coexistence of two attractors, where (average) price and housing stock are higher and lower than their fundamental values, respectively. This is particularly clear from the bifurcation plot of the stock of housing (middle panel). Beyond this range of coexistence, the two attractors merge again into a unique attractor, giving rise to the dynamic patterns represented in the bottom panels (for $\mu=10$ ). Similar bifurcation plots with respect to $\mu$ can be obtained under alternative parameter settings around those used in this example. Moreover, further simulations not reported here show that larger values of the slope of the supply curve are also likely to bring about such situations of coexistence. ${ }^{29}$

\section{*** FIGURE 4 APPROXIMATELY HERE ***}

\section{Conclusions}

Significant boom-bust housing price cycles, which can be quite harmful for the real economy, have repeatedly been observed in the past. According to Shiller $(2005,2008)$, speculative behavior is the main driver of these price dynamics. In this paper, we have thus developed a housing market model in which part of the demand for houses is speculative. Overall, we find that speculation may indeed destabilize otherwise stable housing markets. Moreover, analytical and numerical explorations indicate that there are two different routes which can lead to boom-bust housing price cycles. One route is via a Neimark-Sacker bifurcation. Here, fixed point dynamics first turn into cyclical or quasi periodic motion and then, at least for some parameter combinations, into complex dynamics. A second route is via a pitchfork bifurcation. Here, a unique steady state is accompanied by two additional steady states. Hence, housing markets may be permanently overvalued or undervalued. Moreover, irregular switches between bull and bear markets may also be observed if certain speculative forces become stronger.

Our work may be extended in several directions. Unfortunately, our understanding of the price formation in housing markets is far from being complete. Therefore, we need more theoretical insights into the functioning of housing markets. A group centered around Laura Gardini recently initiated a new research field in which piecewise-smooth or even discontinuous maps are used to study various economic problems (see, for instance, Bischi et al. 2009, Tramontana et al. 2010, Sushko et al. 2010). A similar research effort seems to be worthwhile in the case of housing markets: for instance, in the real world, housing developers tend to stop new constructions if house prices become too low or there is permanent excess supply. Moreover, theoretical contributions in this area should, eventually, be tested empirically. One way to do this is to calibrate these models such that they match some stylized facts of housing markets. Another way is to estimate these models. Compared to agent-based financial market models, however, the poor data availability is still a serious issue in this endeavor. While there are large financial market data sets with thousands of daily observations, data on housing markets is much more limited. Therefore, theoretical papers

\footnotetext{
${ }^{29}$ For instance, under the same parameter setting of Figure 4, coexisting attractors can be numerically observed by means of bifurcation diagrams against parameter $\theta$, for $\mu=8$ and $\theta$ ranging between 0.5 and 0.8 .
} 
may be even more relevant than ever.

\section{Acknowledgements}

This work was carried out with the financial support of MIUR (Italian Ministry of Education, University and Research) within the PRIN Project "Local interactions and global dynamics in economics and finance: models and tools". We are grateful to Carl Chiarella for his comments and suggestions on an earlier draft of the paper.

\section{References}

Agliari, A., Dieci, R. and Gardini, L. (2007): Homoclinic tangles in a Kaldor-like business cycle model. Journal of Economic Behavior and Organization, 62, 324-347.

Bischi, G.I., Gallegati, M., Gardini, L., Leonbruni, R. and Palestrini, A. (2006): Herding behaviours and non-fundamental high frequency asset price fluctuations in financial markets. Macroeconomic Dynamics, 10, 502-528.

Bischi, G.I., Gardini, L. and Merlone, U. (2009): Impulsivity in binary choices and the emergence of periodicity. Discrete Dynamics in Nature and Society, 2009, Article ID 407913.

Brock, W. and Hommes, C. (1997): A rational route to randomness. Econometrica, 65, 10591095

Brock, W. and Hommes, C. (1998): Heterogeneous beliefs and routes to chaos in a simple asset pricing model. Journal of Economic Dynamics Control, 22, 1235-1274.

Case, K. (2010): Housing, land and the economic crisis. Land Lines, 22, 8-13.

Chiarella, C, Dieci, R, and Gardini, L. (2002): Speculative behaviour and complex asset price dynamics: A global analysis. Journal of Economic Behavior and Organization, 49, 173-197.

Chiarella, C., Dieci, R. and Gardini, L. (2005): The dynamic interaction of speculation and diversification. Applied Mathematical Finance, 12, 17-52.

Chiarella, C., Dieci, R. and He, X.-Z. (2009): Heterogeneity, market mechanisms, and asset price dynamics. In: Hens, T. and Schenk-Hoppé, K.R. (eds.): Handbook of Financial Markets: Dynamics and Evolution. North-Holland, Amsterdam, 277-344.

Chiarella, C., and He, X.-Z. (2002): Heterogeneous beliefs, risk and learning in a simple asset pricing model, Computational Economics, 14, 95-132.

Chiarella, C., He, X.-Z. and Hommes, C. (2006): A dynamic analysis of moving average rules. Journal of Economic Dynamics and Control, 30, 1729-1753.

Day, R. and Huang, W. (1990): Bulls, bears and market sheep. Journal of Economic Behavior and Organization, 14, 299-329.

De Grauwe, P. (2010): The scientific foundation of dynamic stochastic general equilibrium (DSGE) models. Public Choice, 144, 413-443.

De Grauwe, P., Dewachter, H. and Embrechts, M. (1993): Exchange rate theory - chaotic models of foreign exchange markets. Blackwell, Oxford.

De Grauwe, P. and Grimaldi, M. (2006): Exchange rate puzzles: a tale of switching attractors, European Economic Review, 50, 1-33. 
Dieci, R. and Westerhoff, F. (2010): Heterogeneous speculators, endogenous fluctuations and interacting markets: a model of stock prices and exchange rates. Journal of Economic Dynamics and Control 34, 743-764.

Dieci, R. and Westerhoff, F. (2011): A simple model of a speculative housing market. Journal of Evolutionary Economics, in press. DOI: 10.1007/s00191-011-0259-8.

Eichholtz, P. (1997): A long run house price index: the Herengracht index, 1628-1973. Real Estate Economics, 25, 175-192.

Eitrheim, Ø. and Erlandsen, S. (2004): House price indices for Norway 1819-2003. In Eitrheim, Ø., Klovland, J. T. and Qvigstad, J. F. (eds.): Historical Monetary Statistics for Norway 1819-2003. Norges Bank Occasional Paper no. 35, 349-375. Norges Bank, Oslo.

Farebrother, R.W. (1973): Simplified Samuelson conditions for cubic and quartic equations. The Manchester School, 41, 396-400.

Glaeser, E., Gyourko, J. and Saiz, A. (2008): Housing supply and housing bubbles. Journal of Urban Economics, 64, 198-217.

Hommes, C., (2001): Financial markets as nonlinear adaptive evolutionary systems. Quantitative Finance, 1, 149-167.

Hommes, C. and Wagener, F. (2009): Complex evolutionary systems in behavioral finance. In: Hens, T. and Schenk-Hoppé, K.R. (eds.): Handbook of Financial Markets: Dynamics and Evolution. North-Holland, Amsterdam, 217-276.

Kindleberger, C. and Aliber, R. (2005): Manias, panics, and crashes: a history of financial crises, 5th ed. Wiley, Hoboken.

Kouwenberg, R. and Zwinkels, R. (2011): Chasing trends in the US housing market. Working Paper, Erasmus University Rotterdam, available at: http://ssrn.com/abstract=1539475.

Leung, A., Xu, J. and Tsui, W. (2009): A heterogeneous boundedly rational expectation model for housing market. Applied Mathematics and Mechanics (Engl. Ed.), 30, 1305-1316.

Lines, M. and Westerhoff, F. (2011): Effects of inflation expectations on macroeconomic dynamics: extrapolative versus regressive expectations. Studies in Nonlinear Dynamics and Econometries, in press.

Lux, T. (2009): Stochastic behavioural asset-pricing models and the stylized facts. In: Hens, T. and Schenk-Hoppé, K.R. (eds.): Handbook of Financial Markets: Dynamics and Evolution. North-Holland, Amsterdam, 161-216.

Medio, A. and Lines, M. (2001): Nonlinear dynamics: A primer. Cambridge University Press, Cambridge.

Parke, W.R. and Waters, G.A. (2007): An evolutionary game theory explanation of ARCH effects. Journal of Economic Dynamics and Control 31, 2234-2262.

Piazzesi, M. and Schneider, M. (2009): Momentum traders in the housing market: survey evidence and a search model. American Economic Review, 99, 406-11.

Shiller, R. (2005): Irrational exuberance (second edition). Princeton University Press, Princeton.

Shiller, R. (2008): The subprime solution. Princeton University Press, Princeton.

Stewart, G.W. (2001): Matrix Algorithms - Volume II: Eigensystems. SIAM, Philadelphia. 
Sushko, I., Gardini, L. and Puu, T. (2010): Regular and chaotic growth in a Hicksian floor/ceiling model. Journal of Economic Behavior and Organization, 75, 77-94.

Tramontana, F., Gardini, L., Dieci, R. and Westerhoff, F. (2009): The emergence of "bull and bear" dynamics in a nonlinear model of interacting markets. Discrete Dynamics in Nature and Society, 2009, Article ID 310471.

Tramontana, F., Westerhoff, F. and Gardini, L. (2010): On the complicated price dynamics of a simple one-dimensional discontinuous financial market model with heterogeneous interacting traders. Journal of Economic Behavior and Organization, 74, 187-205.

Westerhoff, F. (2004): Multiasset market dynamics. Macroeconomic Dynamics, 8, 596-616.

Westerhoff, F. (2009): Exchange rate dynamics: A nonlinear survey. In: Rosser, J.B., Jr. (ed): Handbook of Research on Complexity. Edward Elgar, Cheltenham, 287-325.

Figure Captions

\section{Figure 1}

Dynamics of the 2-D model with speculation $(L=0)$. Base parameters: $\alpha=0.5, \delta=\lambda=0.02$, $\beta=0.05$. Supply parameter $\theta=0.025$. Speculative demand parameters: $\omega=1, \psi=5, \nu=0.01$. Bifurcation diagram of house price $p$ against extrapolation parameter $\gamma$ (top panel) and dynamics of price $p_{t}$ and stock $h_{t}$ for $\gamma=7.3$ (middle and bottom panels, respectively).

\section{Figure 2}

Dynamics of the 2-D model with speculation $(L=0)$. Parameters $\alpha, \delta, \lambda, \beta$ according to our base selection and speculative demand parameters as in Figure 1. Supply parameter $\theta=0.5$. Bifurcation diagram of $p$ against extrapolation parameter $\gamma$ (top panel) and dynamics of $p_{t}$ and $h_{t}$ for $\gamma=7.3$.

\section{Figure 3}

Dynamics of the 3-D model $(L=1)$. Parameters $\alpha, \delta, \lambda, \beta$ according to our base selection. Speculative demand parameters: $\bar{w}=0.5, \psi=5, \kappa=100$. Top panels (supply parameter $\theta=0.5$ ): bifurcation diagram of $p$ against chartist parameter $\mu$ (left) and attractor in the plane $(h, p)$ for $\mu=6.9$ (right). Bottom panels $(\theta=0.025)$ : bifurcation diagrams of house price $p$ (left) and stock $h$ (right) against parameter $\mu$.

\section{Figure 4}

Dynamics of the 4 -D model $(L=2)$. Parameters $\alpha, \delta, \lambda, \beta$ according to our base selection. Speculative demand parameters: $\psi=\widehat{\psi}=0.4, \tau=0.003, K=0$. Supply parameter $\theta=0.5$. Top and middle panels: bifurcation diagrams of $p$ and $h$ against chartist parameter $\mu(=\widehat{\mu})$, respectively. Bottom panels: dynamics of $p_{t}$ (left) and $h_{t}$ (right) for $\mu=10$. 